\title{
Service Delivery Location Telecom Address
}

National Cancer Institute

\section{Source}

National Cancer Institute. Service Delivery Location Telecom Address. NCI Thesaurus.

Code C95400.

The telecom address of the service delivery location. 